\title{
Review on Multiple Ethical Review in North-South Collaborative Research: the Experience of the Ebola-Tx Trial in Guinea
}

\author{
Article by Labban Fredrick Sichivula \\ M.Sc Clinical Research, Texila American University, Zambia \\ Email: labbans25@yahoo.com
}

\section{Introduction}

For the purposes of this paper, the review of the article titled"Multiple ethical review in NorthSouth collaborative research: the experience of the Ebola-Tx trial in Guinea" published in 2016 in the Indian journal of medical ethics, will focus on the following subtitles; the literature review, article summary and structure. In critiquing this article, the author of this paper will explore the credentials and work of the article's author to establish its authority and accuracy. We will deal with the time surrounding the publication of the article as well as the current references cited in the article. The objectivity of the article will be considered in terms of biasness, consideration of holistic information surrounding the subject and how the author navigated through the various issues to remain objective. The article'sstability will be reviewed based on the type of publication. The author will also zero in on the tables to point out important and relevant information. We will also review the relevance of the content in relation to the title of the article. This paper will also point out some of the recent work being done related to multiple ethical reviews in research and at the end of this article review, a clear and concise conclusion will be given.

\section{Review of literature}

Double ethical review is defined as the review of a research proposal involving ethics committees in countries of research site and sponsor. The practice is aimed at minimizing double standard practice and enhance the protection of participants and the population (Ravinetto et al., 2010).

The difference in research standards, research interests and cultural differences precipitate the use of multiple ethical review to harmonise research ethics. Researchers have called for the need for ethical review collaboration between host and sponsor countries (Hyder et al., 2004). They point out that this reflects the desire for focused capacity development in ethical review. They also note the reluctance of somehost (African) IRBs to raise issues to deal with confidentiality, local language consent forms and letter for approval which sponsor countries significantly raised. There are three areas of concern in research done in developing countries that have been identified which may demand double ethical review; these include the standard of care that should be used in clinical trials, reasonable availability of interventions that are proven to be useful during research trials and the quality of informed consents (Emanuel, Wendler, Killen and Grandy 2004). The issues raised by the researchers necessitate cooperating partners to increase capacity building in the protection of research participants. However, some authorities have expressed concern over the use of multiple ethical review that this may cause delay in execution of the study. They reiterate that the process is over burdening and involve a lot of time resources and it duplicates the process (Improved Research Ethics Review Process for Studies done Across Nations 2012).

Most scholars involved in multinational research have advocated for coordinated dialogue among ethical committees in host and sponsor countries so as to harmonise the research ethics. These efforts would provide a platform to resolve conflicting issues and enhance capacity building which will in turn better protect research participants. 
Texila International Journal of Clinical Research

Volume 3, Issue 2, Dec 2016

\section{Article summary}

The purpose of this article aims to review the experiences of multiple ethical reviews in the Ebola treatment trial that was conducted in Guinea during the unprecedented outbreak of the infectious disease in 2014.

The authors outlined the various ethical committees/Institutional review boards in Guinea and European countries which were sponsors involved in the review process. The objective of the research was to assess double ethical review and evaluate the implementation of the World Health Organization background document of September 2014 ("flexible approaches are required to harmonise various review processes, and ensure that the various ethics committees can review the projects simultaneously and share and discuss the review outcomes with each other"). The article points out different recommendations that were made by multiple ethical committees and how they were incorporated in the final research protocol. The author points out that multiple ethical reviews brings out complimentary views that benefit the research participants and enhance acceptability of the research by the community. The researcher however, also highlightsthe conflicting views that were raised during ethical reviews and the challenges to reconcile them in the case of emergency outbreaks where time is a limiting factor. It was recommended that there should be dialogue among various ethics committees to harmonize the review process.

\section{Article structure}

The structure of the article was developed in accordance with the outlined format of the Indian Journal of Medical Ethics. It begins with a title limited to 15 words. The abstract is well presented beginning with a definition of "double ethical review" and objective of the study. It goes on to describe the Ebola-Tx trial protocol approval process on which basis this article stands. The methodology used is not clearly indicated. The author went on to outline the findings of the study, the conclusion and recommendations.

The article continues with a provision of background information regarding the use of double ethical reviews in externally sponsored research. Information on double ethical review in the EbolaTx trial is well described. On the first page, the authors' information box is given. Information on Ebola treatment options and protocol submissions to various ethical groups is given. The objective of the study are clearly outlined. The tables provided in the articles lists members of the Ebola-Tx consortium, ethical committees that reviewed the protocol. The methodology of the study is given in the main body and has provided specifics on what the researchers focused on.

The section for findings contains information on submission requirements that were requested by different ethical committees. It also provides information on the timeliness of the reviews which was crucial in the Ebola-Tx trial as well as a table to illustrate the time lapses from when the submissions were made to how long the ECs took to respond and when amendments where incorporated in the protocol. The contents of the review is included in form of a table and comments.

The discussion section is well formatted and a conclusion section is given. The article ends with acknowledgements, comment on funding and a statement of conflict of interest, notes and references.

\section{Authority}

The qualifications of the author is not given in the article. However, the credibility can be authenticated for the fact that the researcher works with a credible institution in clinical sciences department at the Institute of Tropical Medicine, Antwerp, Belgium. The author is a professional and is subscribed with the Indian journal of Medical Ethics that published the article on $25^{\text {th }}$ January, 2016. The co-authors are indicated to belong to the same institution as well as the other that is involved with Centre National de Formation et Recherche en Santé Rurale de Maferinyah, Conakry, Guinea. The authors were directly involved in the research process of Ebola Treatment trial and the have sound knowledge of the experience alluded to in the article. 


\section{Accuracy}

The information provided in the article is generated from a recent outbreak of Ebola in West Africa that captured the international attention in 2014. This attracted a lot of highly acclaimed world researchers to combat the Ebola virus disease outbreak and their experience is pointed out in the article under review. The article uses recent references and has made reference to several regulatory guidelines both international and local (Guinea). The information in the article can be verified using several media and academic sources in relation to the event that was widely publicized. The documents from World Health Organization is widely used to provide a credible background information on the use of convalescent plasma in Ebola therapy.

\section{Currency}

This is a recent article, published in January 2016. The events surrounding the basis of the research is within a period of two years from the time the Ebola treatment trial and time of publication. The references used range from 2001 to 2015. The post evaluation of the Ebola outbreak and treatment trial is still ongoing which supports that the article is current. The research protocol was first submitted to the ethics committees/institutional review boards on $22^{\text {nd }}$ November, 2014. The final draft of the protocol referred to in the article was approved by several ethical committees between 12 and 26 January, 2015 leading to the first online publication of the article in 2016.

\section{Relevance of the content}

This article was aimed at assessing the concept of multiple ethical reviews in order to make recommendations for research conducted during public health emergencies. It is applicable in the process where research is conducted is one country whereas the sponsors are from another country. The article generates important issues researchers are likely to face in a similar setting. These issues include, acceptability of the research by participants and the community, capacity building for local researchers and most of all, better protection of participants. It is also applicable in resolving conflict of interest between sponsors from another country and the local community where the research is conducted. With enhanced communication among different ethical committees, it would share knowledge and harmonise ethical review processes.

\section{Objectivity}

The author of the article discussed the pros and cons of multiple ethical reviews without bias. The article contains a statement declaring conflict of interest so as to remain as objective as possible. The research is based on lived experiences and supporting guidelines from research regulators. Referencing from other academic literature of different views on multiple ethical review has been exercised to eliminate bias. The conclusion of the researchers in the article points out the conflicting issues that were raised by different ethical review committees and indicates how each issue was resolved to ensure compliance. The recommendations given in the conclusion is in line with harmonization of ethical reviews as outlined by World Health Organization.

\section{Stability}

The article was published online by an establishedinternational academic journal "The Indian Journal of Medical Ethics" Vol I No 2 between April-June of 2016 whose database is stable. The journal has specific guidelines on the criteria of articles they accept and publish. It requires subscription of membership for contributors.

\section{Analysis of table}

The first table given in the article lists the various members of the Ebola treatment consortium. These institutions range from universities, research organizations, regulatory organization and World Health Organization which is the major world body dealing with Health issues. 
Texila International Journal of Clinical Research

Volume 3, Issue 2, Dec 2016

The second table is a list of multiple ethical committees that were involved in the protocol review process. These committees are station in both European and African countries as well as WHO.

The third table indicates different time intervals that the ethics committees took to review the protocol upon submission and for the re-submission of amendments.

The last table is a summary of multiple issues that were raised by the ethical review committees and how the comments were treated to comply with the recommendations.

\section{Recent advances related to the topic}

Scholars in the field of clinical research are working on means that will promote speedy implementation of research especially in public health emergencies like the "Ebola outbreak." Many lessons have been leant over the years on how study delays can be minimized. Schopper et al (2015) have suggested five innovative practices in research; introduction of policy to exempt a posteriori analysis of routinely collected data, preapproval of emergency protocols, general ethical approval of routine surveys, new framework to guide ethics review and evaluating the impact of approved studies. They point out that these measures would enhance the process of research. With the increasing demand for global health training, Crump and Surgarman (2010) point out that there must be guideline in place to foster best ethical practices in training of students who wish to gain experience in different countries. With the current advancement in the medical field of organ transplant Erin and Arris (2003) proposed the ethical support of the organ sale markets. They have suggested the use of a single marked of human organs within a confined market place. In the world of sport, Kayser, Mauron and Miah (2007) suggest that rather than striving for eradication of doping in sports, which appears to be an unattainable goal, a more pragmatic approach aimed at controlled use and harm reduction may be a viable alternative to cope with doping and doping-like behavior. There is a marked underrepresentation of countries in high-impact general medical journals. This has high ethical implications concludes Sumathipala, Siribaddana and Patel (2004). This conclusion resonates with the idea of enhancing capacity building research ethics for researchers in the developing countries. Beeson and Lippman (2006) have expressed concern over the harvesting of multiple eggs [that] often involve the administration of drugs that have not been approved for this purpose. These issues highlighted are meant foster improved research ethics in stem cell research.

\section{Conclusion}

The review of the article has been summarized and critically analyzed. The abstract provides the significance of the study, objective, findings, conclusion and recommendations.

The background outlines the use of double ethical review in multinational studies in situations. In this review, the research was conducted in Guinea while sponsors were from Belgium and other countries. The double ethical review is meant to increase better protection for participants and to harmonize research ethics.

The Ebola-Tx trial involved the interventional use of convalescent plasma in the treatment of Ebola Virus Disease (EVD). The study protocol was submitted to various ethics committees who reviewed and made recommendations that became part of the final protocol. The article was focused on assessing the experience of double ethical review and to make recommendations on this practice in future public health emergencies.

The critical review of this article recognizes the meticulous approach the author used to discuss the merits and demerits of double ethical review. The findings show that multiple ethical review may increase the complexity of the protocol especially when there are conflicting issues raised by the IRBs. The author also points out that the practice increases better protection of participants when ethical committees involved have open dialogue in the review process and promotes capacity building. The findings in the article provides an in-depth insight in the approval of research protocols involving multinational organizations. The host country and sponsors country's research organizations need coordinated efforts to harmonise research ethics as recommended by WHO. 


\section{References}

[1] Beeson, D., \& Lippman, A. (2006). Egg harvesting for stem cell research: medical risks and ethical problems. Reproductive BioMedicine Online, 13(4), 573-579.

[2] Crump, J., Sugarman, J. \& Working Group on Ethics Training (WEIGHT.) (2010). Ethics and best practices guidelines in Global Health. The American Journal of Tropical Medicine and Hygen, 83(6), 1178-1182

[3] Hyde, A.A, Wali, S.A., Khan, A.N., Tech, N. B., Kass, N.E., \& Dawson, L. (2004). Ethical review of Health research; a perspective for developing country researchers. Journal of Medical Ethics, 30(1), 68-72.

[4] htt://globalhealthtrials.tghn.org seen on 14.11.2016.

[5] Erin, C. A., \& Harris, J. (2003). An ethical market in human organs. Journal of medical ethics, 29(3), 137138.

[6] Emanuel, E.J., Wendler, D., Killen, J., \& Gandy, C. (2004). What makes clinical research in developing countries ethical? The benchmark of ethical research. Journal of Infectious Diseases, 189(5), 930-937.

[7] Kayser, B., Mauron, A., \& Miah, A. (2007). Current anti-doping policy: a critical appraisal. BMC medical ethics, 8(1), 1.

[8] Ravinia, R., Tinto, H., Rouamba, A., Talisuna, Adoke, Y., Kadima Ebeja, A., Maketa, V., Grieftens, K.P., Buve, A. \& Crawley, F. (2010). Health research: The challenges related to ethical review and informed consent in developing countries. Giornale Italiano Di Medicina Tropicale, 15.N.1-4, p15.

[9] Sumathipala, A., Siribaddana, S., \& Patel, V. (2004). Under-representation of developing countries in the research literature: ethical issues arising from a survey of five leading medical journals. BMC Medical Ethics, $5(1), 1$.

[10] Schopper, D., Dawson, A., Upsani, R., Ahmad, A., Jesani, A., Ravinetto, R., Segelid, M.J., Sheel, S., \& Sigh, J., (2015). Innovation in research ethics governance in humanitarian settings BMC medical ethics. DOI 1186/5 $12910-015-0002-3$

\section{Source}

De Crop, M., Delamou, A., van Griensven, J., \& Ravinetto, R. (2016). Multiple ethical review in North-South collaborative research: the experience of the Ebola-Tx trial in Guinea. Indian journal of medical ethics, 1(2). Viewed on $28^{\text {th }}$ October 2016.

http://www.issuesinmedicalethics.org/index.php/ijme/article/view/2342 